\title{
MOVEMENT, DISEASE AND PATCH EXPLOITATION IN NESTING AGENT GROUPS
}

\author{
Wayne M. Getz \\ Krti Tallam \\ Depart. ESPM, University of California \\ Berkeley, CA 94720-3114, USA \\ wgetz@berkeley.edu,krtital@gmail.com
}

\author{
Richard Salter \\ Numerus Inc. \\ 850 Iron Point Rd. \\ Folsom, CA 95630, USA \\ richard.salter@numerusinc.com
}

\begin{abstract}
Animal relocation data has recently become considerably more ubiquitous, finely structured (collection frequencies measured in minutes) and co-variate rich (physiology of individuals, environmental and landscape information, and accelerometer data). To better understand the impacts of ecological interactions, individual movement and disease on global change ecology, including wildlife management and conservation, it is important to have simulators that will provide relatively simple demographic, movement, and epidemiology models against which to compare patterns observed in empirical systems. Here we describe a simulator that accounts for the influence of consumer-resource interactions, existence of social groups anchored around a central location, territoriality, group-switching behavior, and disease dynamics on population size. We use this simulator to develop new and reinforce existing results and point out areas for future study.
\end{abstract}

Keywords: Consumer-resource interactions, agent-based models, Numerus Model Builder, SIR models, metapopulations

\section{INTRODUCTION}

Disease ecology is a rich field of study that synthesizes inter alia demographic, epidemic, behavioural, movement, spatial and community processes in an ecological setting (Dougherty et al. 2018, Altizer et al. 2011, Johnson et al. 2015). Thus, understanding the impact of particular diseases on wild populations in ecological settings is a challenge that requires a comparison of the spatial and community structure of these focal populations, as they might arise in the absence versus the presence of the causative agents of the diseases of interest. One way of doing this is to build simulation models that incorporate demographic, spatial, movement, and other relevant behavioral and community processes, and then compare simulations of these models across various scenarios (White et al. 2018) or outcomes with and without epidemiological processes added to the mix. We take this latter approach and our primary aim is to develop a tool that can be used to study the impact of disease on colonial populations and metapopulations (Hanski et al. 1997). This tool must be comprehensive enough to include critical processes needed to address questions that will extend our current knowledge in significant ways. Its application, of course, is limited by the structure of the model. Thus, in building the model, we are guided by principles related to concepts of "appropriate complexity modeling" (Getz et al. 2018, Larsen et al. 2016), which guides methods for extending the model to address questions that require the incorporation of additional processes into the model. The modular model structure imposed by the architecture of the Numerus Model Builder (NMB) platform greatly facilities its modification, including the incorporation of new process (Getz et al. 2015, Getz et al. 2018). 
The standard approach to modeling disease outbreaks caused by directly-transmitted pathogens is through systems of differential or difference equations that model the number or density of susceptible (S), infectious (I), and recovered or removed (R) individuals (Hethcote 2000, Getz and Dougherty 2018). Systems approaches, however, limit our ability to consider individual-level traits, unless these traits can be reasonably well expressed by dividing the population into a limited number of subgroups, such as groups of individuals infected with different strains of pathogens (Keeling and Rohani 2011). Including individual-level variation in disease models requires an agent-based (Grimm et al. 2006) or network approach (Keeling et al. 2010), which then allows both individual behavior and pathogen exposure history to be incorporated into the model. It also allows tracing the number of individuals each pathogen infects (Getz et al. 2015) and, hence, the identification of individuals that may be acting as super-spreaders (Lloyd-Smith et al. 2005).

Here, we develop an agent-based formulation that has application to studying disease outbreaks in wildlife populations whose state of health-and hence, susceptibility - is impacted by various factors, including social factors related to group living and the structure of social networks (Sah et al. 2017), ecological factors related to foraging success, and the movement behavior of individuals. The model also includes a periodic return to a colony or nest cell, which implies that the model has both an individual-level and a group-level (household or colony) structure. Such models have applications in animal production systems (e.g., managing foot and mouth disease, James and Rushton 2002), wildlife conservation (Altizer et al. 2011), and disease management at the human-animal interface (Alexander et al. 2018). The latter involves diseases such as anthrax (Carlson et al. 2018), bovine tuberculosis (De Garine-Wichatitsky et al. 2013), and brucellosis in cattle and wild ungulates (Dean et al. 2012), chronic wasting disease in deer (Williams 2005), and toxoplasmosis in rats, cats and humans (Saadatnia and Golkar 2012).

\section{METHODS}

No single model can incorporate all possible processes and the model we present here, for example, does not include age structure. Of course, it can be elaborated in this direction, should age structure be particularly germane, as it would in populations where different age classes have different levels of susceptibilty to disease (but see Coulson et al. 2001, Diekmann and Heesterbeek 2000). For clarity, we outline belowwithout mathematical details - the processes incorporated in our model, leaving such details to the Technical material that can be found in our supporting online file (SOF). Aspects of the Numerus Model Builder construction of the model and the generated NovaScript (extended JavaScript language) implementation are also provided in this file.

\subsection{Model Outline}

The model is formulated as a set of agents (individuals) moving over a cellular array. The agents themselves can be differentiated with respect to type (e.g., sex), can undergo discrete state transitions (e.g., disease state) and can also change continuously over time with respect to that state of each agent, such as in our case, its current "mass" (also a surrogate for fitness) and its current "stress level" (which accumulates when the individual is unable to meet its resource needs each time period). The cells in the cellular array may also have relatively complex states that, in our model, include a list of the agents in each cell and the level of a dynamically responding resource, which determines the environmental context for the agents. The movement of each agent over the landscape is assumed to be influenced by reproductive, ecological, and epidemiological processes but not on colony membership or other agent factors. More specifically, in our model, the following objects and concepts apply (mathematical and coding details provided in the SOF):

Simulation world. This is the set of agents moving over a cellular array (in our case hexagonal and with a toroidal topology). 
Cells. These are spatial extents (or patches) that contain resources, host at most one colony site, and can be occupied by one or more agents who either pass through in a single time period or remain for some time during which they exploit the dynamically changing (through both growth and extraction) resources.

Time. The action takes place over discrete time steps during which individuals (agents) move through one or more cells, stay in a cell to exploit resources, or return to their colony cell at regular intervals of time where they may reproduce.

Agents. Each agent has a biomass (fitness surrogate), a stress level, and a sex determined at birth. Females may reproduce on regular returns back to their colonies by, essentially, fissioning into two.

Agent association groups (colonies). Each agent belongs to one of several association groups called colonies. These colonies have a home or nest cell. An agent belonging to a particular colony marks each cell that it moves through with a colony-specific marker. The accumulation of such markers, with fading effects over time, facilitates the emergence of territorial movement behavior.

Consumer-resource ecology. When individuals move they loose biomass, but if they remain in a cell they gain biomass. Changes in biomass are computed using dynamic consumer-resource interaction processes that incorporate stress components as well (Getz 2011). These dynamics account for resource growth, saturation, storage processes, and agent resource-extraction rates.

Territoriality. Cells have a fading memory of the accumulated visits of all individuals from a particular colony through the deposit of a scent-like marker each time an agent enters the cell.

Movement. At each time-step-except when moving back to the colony at regular intervals of timeindividuals move to cells, selected with given probabilities based on their relative attractiveness. This attractiveness increases with resource richness and decreases with distance, as well as with the strength of the markings of agents from foreign colonies.

Colony switching (fission-fusion dynamics). In some scenarios we allow individuals to switch membership of their current colony when they enter a cell that is the nest location of a foreign colony. The specific rule we use, when "colony switching" is permitted, is for individuals to switch colonies with probability 0.8 if the foreign colony in question is no more than $30 \%$ as large as their current colony.

Births and deaths. Only females reproduce with a particular probability at regular nest-cell return times, provided they are sufficiently fit (i.e., large) and not too stressed. Individuals die at a naturalmortality specified rate, plus an additional disease-induced-mortality rate during their infectious period.

Force of disease transmission. Each cell presents a risk of infection to susceptible (S) agents entering that cell with a force proportional to the number of infected (I) individuals currently in the cell added to a fading value of past visits by infected individuals. This incorporation of accumulated but fading risk from past visits allows the model to deal with both direct (current I individual visits) and indirect disease transmission (past visits of I individuals),

Epidemiology. We allow individuals to cycle through an $\mathrm{S}$ (susceptible), E (exposed but not yet infectious), I (infectious), V (immune), S cycle at rates respectively proportional to the risk force of disease transmission, the inverse of the latent period, the inverse of the infectious period, and the inverse of refractory immune period. Individuals in all stages are subject to a natural mortality rate, while individuals in the I stage are subject to an additional disease-induced mortality rate.

We used the above concepts to formulate our equations and develop our simulation code using the Numerus Model Builder (NMB) platform, which greatly facilitates rapid, accurate construction of the model through NMB's hierarchical architecture and chip design. More specifically, cell equations and agent equations were first constructed as individual modules, which were then used to populate a "world" with an openended number of agents moving over an $18 \times 18$ hexagonal cellular array that contained 9 regularly spaced nest cells (Fig. 1). A toroidal topology was used to avoid boundary effects—but, of course, if boundary effects are important or the focus of an investigation, then the NMB allows them to be incorporated once 
equations describing how individuals move at boundaries are included in the code. The simulation period was 2000 time steps, with individuals returning every 10 time steps to their colonies to reproduce.

Some of the details on how to build such models using NMB have been provided elsewhere (Getz et al. 2018, Getz et al. 2015), with additional novel features discussed in our SOF. Notably, the NMB platform has two independent components. The first is a set of coding tools accessed through a graphical user interface (GUI) that is used to generate a NovaScript, which is an enriched JavaScript language. This script can then be run in any suitable run-time environment, where NMB currently provides a Java runtime engine implementable in Windows, MacOS, and Linux environments. An example of a script created for our model is provided in our SOF, together with a table that facilitates moving between the code and the mathematical description provided therein. Finally, our model is available online (see our SOF) and interested readers can run the model after downloading a free version of the NMB platform at the Numerus website.

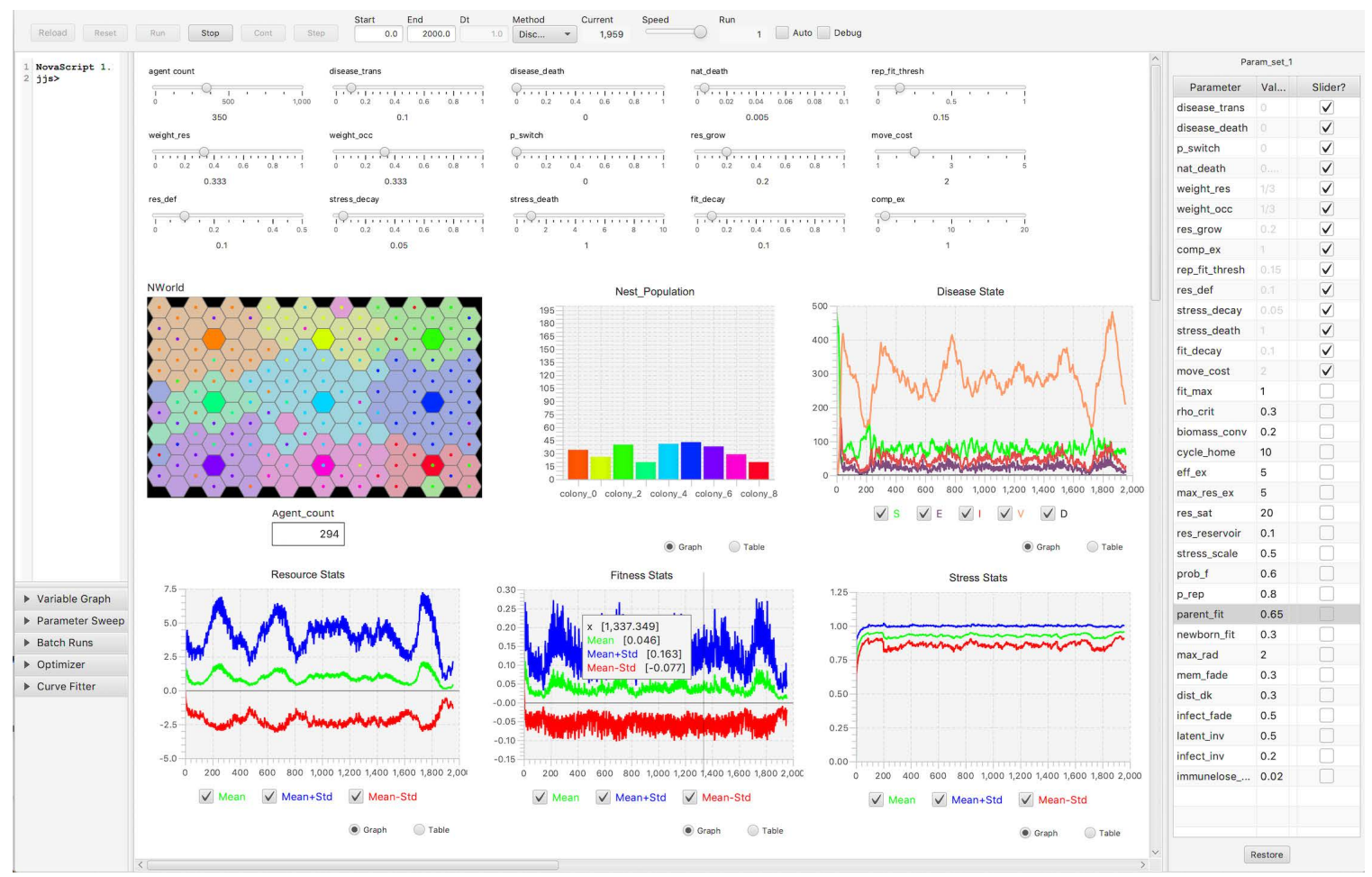

Figure 1: A view of the Numerus model builder (NMB) dashboard that includes output from a simulation run, plotted up to time $t=1959$. Parameters that can be manipulated using sliders appear above the 6 graphics panels, while a full list of parameters and values appear on the right-hand side. The top left graphic panel depicts the $18 \times 18$ hexagonal landscape: colony nest cells are indicated using fully saturated colors their territory cells use partially-saturated corresponding color (note "boundary spillover" effects due to the toroidal landscape topology). The number of individuals in each colony at time $t=1959$ are plotted in the central bar graph, using matching colony nest-cell colors. The number of agents in each disease class are plotted in the upper right-most graphics panel. Plots are also shown of the average cell resource (bottom left graphics panel), agent biomass/fitness (bottom center graphics panel) and agent stress (bottom right graphics panel) levels over time. A mouse-controlled arrow can be used to read particular values from the plotted output (bottom center graphics panel). 
Getz, Salter, and Tallam

\section{RESULTS}

Prior experience with the consumer-resource component of the model (Getz et al. 2015, Getz et al. 2016) and some experimentation led us to select a set of resource and consumer growth and interaction process rate parameters, as well as consumer stress and mortality process rate parameters (Tables $2 \& 3$ in SOF) that were used to generate a baseline set of simulation results against which we could compare the agent and colony population dynamics under different movement and disease scenarios. We set up our NMB dashboard so that we could observe plots of information in real time and create a visual record of all parameter values used to produce the results of interest. An example of this output obtained during one of our simulations is provided in Fig. 1.

\subsection{No disease scenarios}

We ran two instances of the baseline scenario (no disease, no colony switching, weak competition) and obtained similar results with some stochastic variation across runs. A plot of the number of agents $N_{t}$ over time (all are in the susceptible disease state) for the the two runs is provided in Fig. 2A and can are seen to be close in both period and amplitude. We note that our baseline scenario places us at a particular point in the consumer-resource growth-rate, extraction, and interaction parameter value space of our model. Different behaviors are expected at other points in this space, just as a different qualitative behaviors arise in the Lotka-Volterra prey-predator model with the type of functional response that we use in our model (i.e., Beddington-DeAngelis extended to an agent-based setting, as in Cantrell and Cosner 2001, Getz 2013). For example, if we decrease the mutual interference level from 1 (resource extraction inefficiencies due to self and others are proportional to the total biomass of all agents in cells) to 0 (inefficiencies arise only from self) or increase it to 10 (inefficiencies increase ten fold for each competitor in the cell, presumably due to territorial conflicts) then we see a small decrease in the oscillation frequency in the former case (compare plots Fig. 2A with orange plot in Fig. 2B), but a very different type of trajectory in the strong mutual interference case (compare plots in Fig. 2A with orange plot in Fig. 2C). When colony switching is included (compare the blue "switch" and orange "no-switch" population trajectories in Figs. 2B and C) the effects vary depending on the level of competition: a clear effect of colony switching only emerges once extinction rates are considered, as reported below.

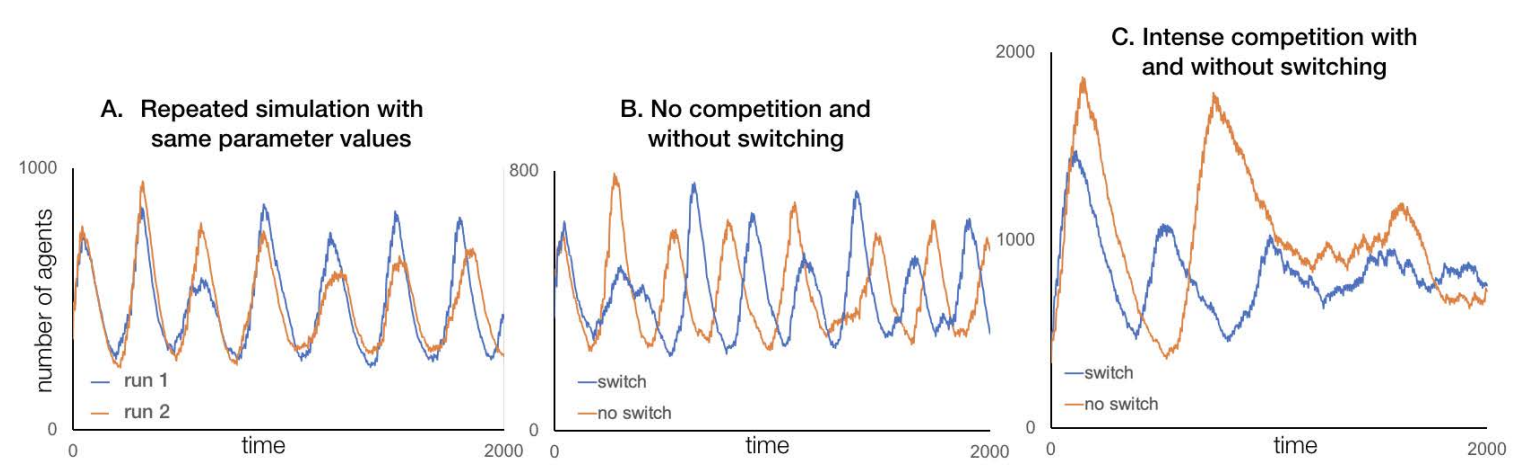

Figure 2: The number of agents $N_{t}$, obtained from simulations of the model, are plotted over the 2000 step time interval: A. two different instances for the same the baseline case (no competition, no colony switching, no disease); B. two simulations without competition $(q=0)$; C. two simulations with competition $(q=10)$. In the latter two cases, no-colony-switching ( $p_{\text {switch }}=0.0$ is represented by the blue plots and colony switching $\left(p_{\text {switch }}=0.8\right)$ by the orange plots. Note that the vertical scales on the panels $\mathbf{A}$ to $\mathbf{C}$ are different. 

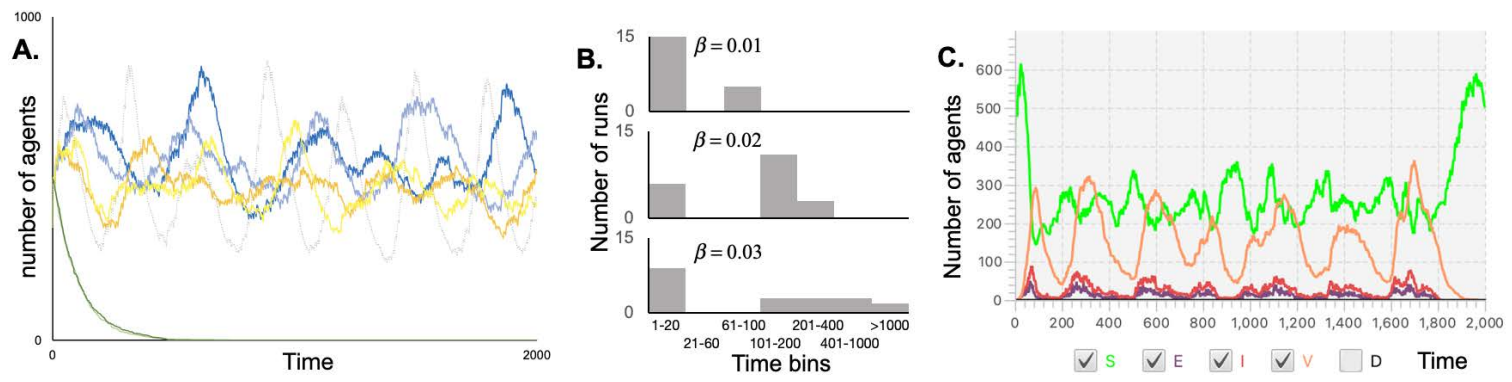

Figure 3: A. The number of agents $N_{t}$ is plotted for the baseline case except for the following modifications to movement behavior. Movement is driven by: territory size maximization (two blue plots); available resource maximization (two green plots); movement cost minimization (orange and yellow plots); and equal weightings of above three (dotted grey plot-the same as blue plot in Fig. 2A). B. The times taken for all infected (I) and exposed (E) individuals to be extirpated by natural mortality (disease mortality rates are zero in these simulations) are plotted as a histogram for 15 replicate simulations for each of the three transmission parameter cases labeled $\beta=0.01,0.2$, and 0.03 (note: time scale is log-like). C. Plots of the number of individuals in disease states $\mathrm{S}, \mathrm{E}, \mathrm{I}$, and $\mathrm{V}$ (immune) for Run 3 of the case $\beta=0.03$, where final extirpation of the epidemic occurred at time $t=1816$ (note that number of dead individuals in state $\mathrm{D}$ (dead) was not plotted since it is integrated — and hence steadily increasing—rather than an instantaneous value of time).

\subsection{Effects of different movement strategies}

We evaluated the effects of our agents moving on the total number of agents over time for the following five strategy rules (note: the rules apply to staying in the current cell or moving to one of 18 cells within a radius of 2 hexagonal rings for all times steps except $t \bmod T_{f}$ when all individuals must be back in their nest cells):

1. maximize available resources

2. maximize territory size

3. minimize movement costs

4. maximize an equally weighted combination of the above three

5. maximize an equally weighted combination of all three, with colony switching (see Section 2.1)

The number of agents produced by an illustrative simulation for each of movement strategies 1-4 are graphed in Fig. 3A. Simulations involving movement strategy 5 were undertaken in the context of exploring the effects of mortality on population viability.

\subsection{Mortality, colony switching and extinction}

To illustrate the impact of mortality rates on population viability, we ran our baseline scenario 15 times with the natural mortality increased from 0.005 to 0.02 . The average extirpation time over these 15 runs was 425 (Table 3, SOF). We then compared this result with 15 runs in which individuals are allowed to switch colonies with a probability of 0.8 whenever they entered a nest cell of another colony that was no more than $30 \%$ as large as their own colony (Table 3, SOF). In this case, the average extirpation time was 587, which is significant larger than the no-switch case at the $p<0.01$ level. 
Getz, Salter, and Tallam

\subsection{Pathogen transmission}

The number of infected individuals in a population, after seeding the population with an exposed (or infectious) individual under non-zero pathogen transmission conditions, follows a U- or J-shaped bimodal distribution (Fig. 3B) that represents failed or short-lived/stuttering outbreaks (Lloyd-Smith et al. 2009). Plots in Fig. 3C represent successful outbreaks (i.e., the number of infected individuals rises to a peak and then falls to zero) - unless endemic conditions ensue (i.e., the presence of infected individuals persists over time, Lloyd-Smith et al. 2005). In the latter case, the bimodal distribution is essentially a long-tailed distribution, given that all stochastic population processes ultimately go to zero, if zero is an absorbing state (which it is in our model since no new infectious individuals are recruited from outside of our metapopulation, Méléard et al. 2012, Fig. 3C). Our model is initially seeded for potential disease outbreaks in that one of the agents at simulation initialization is in disease state $\mathrm{E}$ (exposed), while the remaining 349 are in disease state $\mathrm{S}$ (susceptible). To set up potential pathogen transmission outbreak scenarios requires that the transmission parameter be positive (i.e., $\beta>0$, see Table 1 in the SOF). To explore the nature of these bimodal distributions, we simulated 15 instances each of disease dynamics for the cases $\beta=0.01,0.2$, and 0.03 . The amount of time it took for the number of exposed and infected individuals to fall to zero in each of these cases is represented in the histograms plotted in Fig. 3B. The agent dynamics in the longest-lasting of these 45 runs is illustrated in Fig. 3C. In these simulations, we note that the only effect of pathogens on hostpopulation dynamics is through the assumption that individuals in disease states $\mathrm{E}$ and I do not reproduce, and not through disease-induced mortality (considered next).

\subsection{Disease virulence}

In standard SIR epidemic models (S: susceptible, I: infectious, R: recovered with immunity), the virulence of a pathogen is assumed to be captured by the size of the disease-induced mortality rate parameter. In our model, this parameter is $\mu_{\mathrm{I}}$ (Table $1, \mathrm{SOF}$ ). We investigated the effects of increasing the value of this virulence parameter in the context of a pathogen transmission rate of $\beta=0.05$ (Table 1 , SOF) that leads to outbreaks that generally persist over our 2000 time step interval (cf. Fig. 3B. and C. where for $\beta=$ 0.0314 runs led to extirpation of disease within 1000 time steps and one at time $t=1816$ ). In this case, the number of infectious individuals remained positive for the full simulation interval of 2000 time steps (Fig. 4). As virulence levels increased, the number of infectious individuals decreased and the total number of susceptible individuals increased. The highest virulence level, the number of infected individuals kept being driven down to almost zero, at which which point the number of susceptible individuals increases only to be driven down at the next outburst in the number of infectious individuals.

\section{DISCUSSION AND CONCLUSION}

The results we obtained are meant to provide exemplars of how simulations can be designed to develop quantitative narratives that have the potential to contribute to our understanding of the impact of disease on spatially structured populations that have particular social structures. The model, as constructed, provides a tool for consumer-resource interactions to be explored in the context of individuals moving over a landscape and competing locally (i.e., in cells) to extract a renewable resource, where the extraction function has a Beddington-DeAngelis mutual interference form (Getz 2013). This consumer-resource interaction component has been extensively explored in the context of aggregated population level models that have been extended to include diffusion (Chen and Wang 2005) and viral disease (Elaiw and Azoz 2013) as well. It has not been explored in the context of nesting colonies, however, and so cannot be compared to the results we obtain here. 


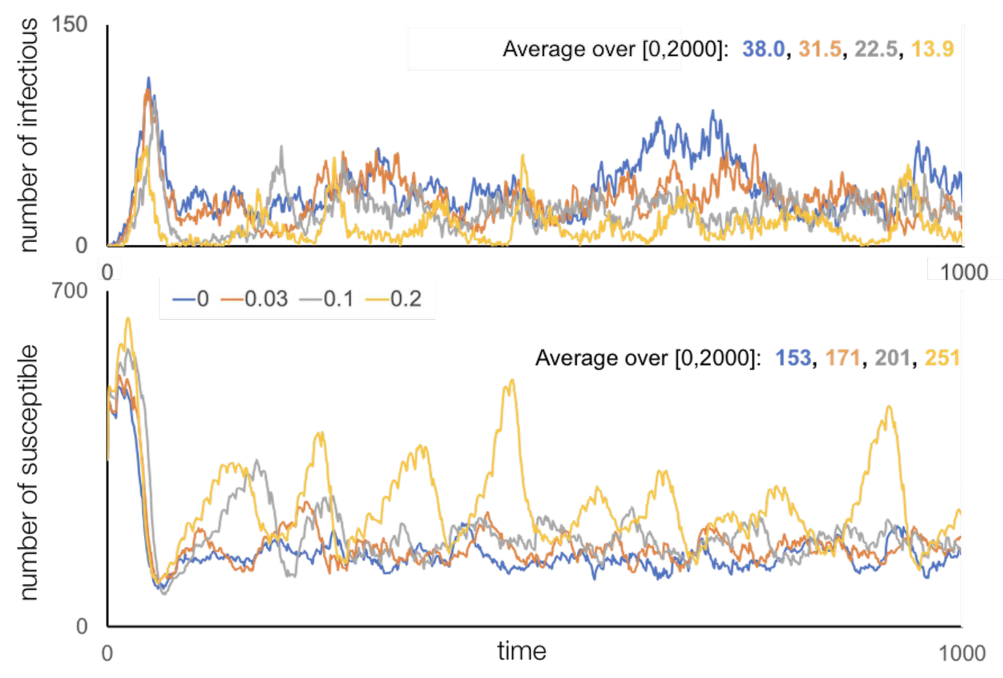

Figure 4: The number of infectious (I: top) and susceptible (S: bottom) agents in four simulations over the interval $[0,2000]$ are plotted here over the first 1000 time steps only for the baseline set of parameter values, except for transmission $(\beta=0.05)$ and disease-induced mortality $\left(\mu_{\mathrm{I}}=0.0\right.$, blue; $\mu_{\mathrm{I}}=0.3$, orange; $\mu_{\mathrm{I}}=0.1$, grey; and $\mu_{\mathrm{I}}=0.2$, yellow). The average values were calculated over the full 2000 time steps.

Agent-based versions of prey-predator processes have been built using the Netlogo (Thierry et al. 2015) and Starlogo (Karsai et al. 2016) modeling platforms. The first of these modeled predation at a "fast" minute-by-minute time scale. Predation events were calculated based on correlated random walks and accounting for predator perceptual ranges and prey refugia. Predation events were then accumulated along with births and deaths into a Lotka-Volterra-type population process iterated at a "slow" daily time scale. This study found inter-alia that increasing predator efficiency by either increasing their perceptual range or decreasing the number of prey refugia resulted in a coexistence region-an equilibrium around which the prey and predatory populations oscillated - decreased for both prey and predator, thereby increasing the risk of extinction.

One result that emerges from simulations of our model is that competition causes the period of prey-predator oscillations to increase and be dampened more rapidly, with this increase and dampening accentuated as colony mixing increases. A second result is that a "grass is greener syndrome" (i.e., movement decisions are based purely on resource considerations) leads to increased stress in individuals and, as a possible result, to population collapse because individuals are not taking into account the cost of moving (green plots in Fig. 3). This collapse (green plots Fig. 3) is mitigated by territoriality considerations, since these curtail movement and allow more time for resource extraction (blue plots in Fig. 3); but movement based on territoriality-only considerations leads to dampening, albeit less so than movement-cost-only considerations (compare yellow with blue plots in Fig. 3). Notably, a movement strategy that accounts for all three factors provides the most regular, albeit oscillatory, population trajectories (dotted grey plot in Fig. 3).

A third result that emerges from our simulations relates to the effects of disease on population dynamics, for which our model provides an appropriate formulation to address the questions of interest. One of these effects is the impact of increasing pathogen transmission rates on the maintenance of an endemic infection. No subclass of a population (e.g., infectious individuals or even the population as a whole) can persist forever-both are bounded stochastic population processes that are known to go extinct in a finite amount of time (Ball and Nåsell 1994, Gordillo et al. 2008). This is amply demonstrated in our simulations that consider the impacts of increasing pathogen transmission rates. In Fig. 3B, we verify that our infection process produces the expected U-shaped bimodal distributions of infectious individuals, though the breadth 
of the distribution associated with the outbreak mode (as opposed to an early-fade-out mode) increases with $\beta$. Indeed, for $\beta=0.05$, once an outbreak occurs, it generally lasts longer then 1000 time steps, as illustrated in Fig. 4. This remains true as the virulence of the pathogen (expressed in the model in terms of the level of disease induced mortality in the host) increases from $\mu_{\mathrm{I}}=0$ (blue plots in Fig. 4) through $\mu_{\mathrm{I}}=0.03$ (orange plots in Fig. 4) and $\mu_{\mathrm{ID}}=0.1$ (grey plots in Fig. 4) to $\mu_{\mathrm{I}}=0.2$ (yellow plots in Fig. 4). What is clear from these simulations is that as virulence increases, the number of infectious individuals decreases, though in our highest virulence case we obtain a sequence of fade outs to near extirpation, followed by outbreaks that are then contained by the high mortality rates of infectious individuals and the transition of those individuals surviving the infection to a state of temporary immunity. This immunity once lost creates, along with births, a rising group of susceptible individuals. Such outbreaks are similar to what we see in seasonal influenza-although, in this case, loss of immunity is hastened by the existence of different influenza strains and mutations in the influenza virus antigens (Lofgren et al. 2007).

A deeper exploration of the various issues examined in this paper, as well as comprehensive investigations of the many other questions that could be asked along the lines of those we posed, would require much more extensive simulations than has been reported here. This is particularly true of simulations involving evaluations of times-to-extinction of populations (Ovaskainen and Meerson 2010), consequences of different movement strategies on the formation and stability of territories (Giuggioli and Kenkre 2014), extirpation of disease outbreaks (Lloyd-Smith et al. 2009) because of the stochastic nature of the processes involved. In the context of developing quantitative narratives, a point is reached where additional simulations will not add to the qualitative character of the information obtained and the increased precision that comes from additional runs is not useful. In other words, there is little value in being more precise than the level of the errors associated with the fit of models to data.

The model we present here has the potential to address a range of questions relating to the ecology of consumer-resource interactions played out over spatially-structured landscapes and impacted by movement and disease processes. Our model can also be elaborated to address questions that include, for example, mating and genetic structures, multispecies situations, environmental factors that exhibit seasonal variation, and landscape structures, including individuals moving over real landscapes. Numerus Model Builder has the facility to import landscape information from GIS databases that can be easily extended to metapopulation settings (Getz et al. 2017); thus, our model can be readily extended to ask questions about spatiallystructured populations on real landscapes.

Of course, considerable interest exists in fitting models to data, especially in the context of wildlife management (McLane et al. 2011) and predicting the behavior of ecological systems that, for example, include a sociological component (Filatova et al. 2013). Agent-based models provide the best, if not the only, approach to addressing many of the more complex questions relating to the management of real socioecological systems and to the advancement of ecological understanding needed to anticipate and reduce the impacts of Covid-2019 type zoonotic outbreaks (Evans et al. 2020).

\section{ACKNOWLEDGEMENTS}

The development of NOVA, a precursor to Numerus Model Builder, was supported by NSF grant CNS0939153 to Oberlin College (PI: RS) and NSF-EEID grant 1617982 (PI: WMG). We thank John Pataki of Logical Laboratories for his considerable help and input into creating and supporting the Numerus Model Builder website http://www.numerusinc.com/. 
Getz, Salter, and Tallam

\section{APPENDIX: SUPPORTING ONLINE FILE}

The supporting online file (SOF) containing details of the mathematical model, the Numerus Model Builder (NMB) construction of our model and a copy of the NovaScript file (superset of JavaScipt and implemented in a Java Run Time environment), is available at https://github.com/wmgetz/SOF/blob/master/ AgentMoveDiseaseSuppInfo.pdf. The NMB model itself can be downloaded at https://github.com/wmgetz/ NMB/blob/master/GetzEtAlOct2019.nmd

\section{REFERENCES}

Alexander, K. A., C. J. Carlson, B. L. Lewis, W. M. Getz, M. V. Marathe, S. G. Eubank, C. E. Sanderson, and J. K. Blackburn. 2018. "The Ecology of Pathogen Spillover and Disease Emergence at the HumanWildlife-Environment Interface”. In The Connections Between Ecology and Infectious Disease, pp. 267298. Springer.

Altizer, S., R. Bartel, and B. A. Han. 2011. "Animal migration and infectious disease risk”. science vol. 331 (6015), pp. 296-302.

Ball, F., and I. Nåsell. 1994. "The shape of the size distribution of an epidemic in a finite population". Mathematical biosciences vol. 123 (2), pp. 167-181.

Cantrell, R. S., and C. Cosner. 2001. "On the dynamics of predator-prey models with the BeddingtonDeAngelis functional response". Journal of Mathematical Analysis and Applications vol. 257 (1), pp. 206-222.

Carlson, C. J., W. M. Getz, K. L. Kausrud, C. A. Cizauskas, J. K. Blackburn, F. A. Bustos Carrillo, R. Colwell, W. R. Easterday, H. H. Ganz, P. L. Kamath et al. 2018. "Spores and soil from six sides: interdisciplinarity and the environmental biology of anthrax (Bacillus anthracis)". Biological Reviews vol. 93 (4), pp. 1813-1831.

Chen, W., and M. Wang. 2005. "Qualitative analysis of predator-prey models with Beddington-DeAngelis functional response and diffusion”. Mathematical and Computer Modelling vol. 42 (1-2), pp. 31-44.

Coulson, T., E. A. Catchpole, S. D. Albon, B. J. Morgan, J. Pemberton, T. H. Clutton-Brock, M. Crawley, and B. T. Grenfell. 2001. "Age, sex, density, winter weather, and population crashes in Soay sheep". Science vol. 292 (5521), pp. 1528-1531.

De Garine-Wichatitsky, M., A. Caron, R. Kock, R. Tschopp, M. Munyeme, M. Hofmeyr, and A. Michel. 2013. "A review of bovine tuberculosis at the wildlife-livestock-human interface in sub-Saharan Africa". Epidemiology \& Infection vol. 141 (7), pp. 1342-1356.

Dean, A. S., L. Crump, H. Greter, E. Schelling, and J. Zinsstag. 2012. "Global burden of human brucellosis: a systematic review of disease frequency". PLoS neglected tropical diseases vol. 6 (10), pp. e1865.

Diekmann, O., and J. A. P. Heesterbeek. 2000. Mathematical epidemiology of infectious diseases: model building, analysis and interpretation, Volume 5. John Wiley \& Sons.

Dougherty, E. R., D. P. Seidel, C. J. Carlson, O. Spiegel, and W. M. Getz. 2018. "Going through the motions: incorporating movement analyses into disease research". Ecology letters vol. 21 (4), pp. 588-604.

Elaiw, A., and S. Azoz. 2013. "Global properties of a class of HIV infection models with BeddingtonDeAngelis functional response". Mathematical Methods in the Applied Sciences vol. 36 (4), pp. 383394.

Evans, T. S., Z. Shi, M. Boots, W. Liu, K. J. Olival, X. Xiao, S. Vandewoude, H. Brown, J.-L. Chen, D. J. Civitello et al. 2020. "Synergistic China-US Ecological Research is Essential for Global Emerging Infectious Disease Preparedness". EcoHealth, pp. 1-14. 
Filatova, T., P. H. Verburg, D. C. Parker, and C. A. Stannard. 2013. "Spatial agent-based models for socioecological systems: challenges and prospects". Environmental modelling \& software vol. 45, pp. 1-7.

Getz, W., O. Muellerklein, R. Salter, C. Carslon, A. Lyons, and D. Seidel. 2017. "A web app for population viability and harvesting analyses". Natural Resource Modeling vol. 30 (2), pp. e12120.

Getz, W. M. 2011. "Biomass transformation webs provide a unified approach to consumer-resource modelling". Ecology letters vol. 14 (2), pp. 113-124.

Getz, W. M. 2013. "Computational population biology: linking the inner and outer worlds of organisms". Israel journal of ecology \& evolution vol. 59 (1), pp. 2-16.

Getz, W. M., and E. R. Dougherty. 2018. "Discrete stochastic analogs of Erlang epidemic models". Journal of biological dynamics vol. 12 (1), pp. 16-38.

Getz, W. M., J.-P. Gonzalez, R. Salter, J. Bangura, C. Carlson, M. Coomber, E. Dougherty, D. Kargbo, N. D. Wolfe, and N. Wauquier. 2015. "Tactics and strategies for managing Ebola outbreaks and the salience of immunization". Computational and mathematical methods in medicine vol. ID 736507, pp. 9.

Getz, W. M., C. R. Marshall, C. J. Carlson, L. Giuggioli, S. J. Ryan, S. S. Romañach, C. Boettiger, S. D. Chamberlain, L. Larsen, P. D'Odorico et al. 2018. "Making ecological models adequate". Ecology letters vol. 21 (2), pp. 153-166.

Getz, W. M., R. Salter, A. J. Lyons, and N. Sippl-Swezey. 2015. "Panmictic and clonal evolution on a single patchy resource produces polymorphic foraging guilds". PloS One vol. 10 (8), pp. e0133732.

Getz, W. M., R. Salter, O. Muellerklein, H. S. Yoon, and K. Tallam. 2018. "Modeling epidemics: A primer and Numerus Model Builder implementation". Epidemics vol. 25, pp. 9-19.

Getz, W. M., R. Salter, D. P. Seidel, and P. Van Hooft. 2016. "Sympatric speciation in structureless environments". BMC evolutionary biology vol. 16 (1), pp. 50.

Giuggioli, L., and V. Kenkre. 2014. "Consequences of animal interactions on their dynamics: emergence of home ranges and territoriality". Movement ecology vol. 2 (1), pp. 20.

Gordillo, L. F., S. A. Marion, A. Martin-Löf, and P. E. Greenwood. 2008. "Bimodal epidemic size distributions for near-critical SIR with vaccination". Bulletin of Mathematical Biology vol. 70 (2), pp. 589-602.

Grimm, V., U. Berger, F. Bastiansen, S. Eliassen, V. Ginot, J. Giske, J. Goss-Custard, T. Grand, S. K. Heinz, G. Huse et al. 2006. "A standard protocol for describing individual-based and agent-based models". Ecological modelling vol. 198 (1-2), pp. 115-126.

Hanski, I., M. E. Gilpin, and D. E. McCauley. 1997. Metapopulation biology, Volume 454. Elsevier.

Hethcote, H. W. 2000. “The mathematics of infectious diseases”. SIAM Review vol. 42 (4), pp. 599-653.

James, A. D., and J. Rushton. 2002. "The economics of foot and mouth disease". Revue scientifique et technique-office international des epizooties vol. 21 (3), pp. 637-641.

Johnson, P. T., J. C. De Roode, and A. Fenton. 2015. "Why infectious disease research needs community ecology". Science vol. 349 (6252), pp. 1259504.

Karsai, I., E. Montano, and T. Schmickl. 2016. "Bottom-up ecology: an agent-based model on the interactions between competition and predation". Letters in Biomathematics vol. 3 (1), pp. 161-180.

Keeling, M. J., L. Danon, M. C. Vernon, and T. A. House. 2010. "Individual identity and movement networks for disease metapopulations". Proceedings of the National Academy of Sciences vol. 107 (19), pp. 88668870 .

Keeling, M. J., and P. Rohani. 2011. Modeling infectious diseases in humans and animals. Princeton University Press. 
Larsen, L. G., M. B. Eppinga, P. Passalacqua, W. M. Getz, K. A. Rose, and M. Liang. 2016. “Appropriate complexity landscape modeling". Earth-science reviews vol. 160, pp. 111-130.

Lloyd-Smith, J. O., P. C. Cross, C. J. Briggs, M. Daugherty, W. M. Getz, J. Latto, M. S. Sanchez, A. B. Smith, and A. Swei. 2005. "Should we expect population thresholds for wildlife disease?". Trends in ecology \& evolution vol. 20 (9), pp. 511-519.

Lloyd-Smith, J. O., D. George, K. M. Pepin, V. E. Pitzer, J. R. Pulliam, A. P. Dobson, P. J. Hudson, and B. T. Grenfell. 2009. "Epidemic dynamics at the human-animal interface". Science vol. 326 (5958), pp. 1362-1367.

Lloyd-Smith, J. O., S. J. Schreiber, P. E. Kopp, and W. M. Getz. 2005. "Superspreading and the effect of individual variation on disease emergence". Nature vol. 438 (7066), pp. 355.

Lofgren, E., N. H. Fefferman, Y. N. Naumov, J. Gorski, and E. N. Naumova. 2007. "Influenza seasonality: underlying causes and modeling theories". Journal of virology vol. 81 (11), pp. 5429-5436.

McLane, A. J., C. Semeniuk, G. J. McDermid, and D. J. Marceau. 2011. "The role of agent-based models in wildlife ecology and management”. Ecological Modelling vol. 222 (8), pp. 1544-1556.

Méléard, S., D. Villemonais et al. 2012. "Quasi-stationary distributions and population processes”. Probability Surveys vol. 9, pp. 340-410.

Ovaskainen, O., and B. Meerson. 2010. "Stochastic models of population extinction". Trends in ecology \& evolution vol. 25 (11), pp. 643-652.

Saadatnia, G., and M. Golkar. 2012. "A review on human toxoplasmosis". Scandinavian journal of infectious diseases vol. 44 (11), pp. 805-814.

Sah, P., S. T. Leu, P. C. Cross, P. J. Hudson, and S. Bansal. 2017. "Unraveling the disease consequences and mechanisms of modular structure in animal social networks". Proceedings of the National Academy of Sciences vol. 114 (16), pp. 4165-4170.

Thierry, H., D. Sheeren, N. Marilleau, N. Corson, M. Amalric, and C. Monteil. 2015. "From the LotkaVolterra model to a spatialised population-driven individual-based model". Ecological modelling vol. 306, pp. 287-293.

White, L. A., J. D. Forester, and M. E. Craft. 2018. "Disease outbreak thresholds emerge from interactions between movement behavior, landscape structure, and epidemiology". Proceedings of the National Academy of Sciences vol. 115 (28), pp. 7374-7379.

Williams, E. 2005. “Chronic wasting disease”. Veterinary pathology vol. 42 (5), pp. 530-549.

\section{AUTHOR BIOGRAPHIES}

WAYNE M. GETZ is a Professor in the Graduate Division at the University of California, Berkeley, and a Founding Partner of Numerus Inc. His email address is wgetz@ berkeley.edu.

RICHARD SALTER is Emeritus Professor of Computer Science at Oberlin College and a Founding Partner of Numerus Inc. His email address is richard.salter@numerusinc.com.

KRTI TALLAM has a B.S. in Statistics and in Natural Resources and Environmental Sciences from the University of Illinois, Urbana-Champaign, and is currently a Fulbright Scholar working on a project in India. Her email is krtital@gmail.com. 